Präv Gesundheitsf 2023 · 18:68-77

https://doi.org/10.1007/s11553-022-00932-6

Eingegangen: 26. Mai 2021

Angenommen: 4. Januar 2022

Online publiziert: 21. Februar 2022

(c) Der/die Autor(en) 2022
Maxi A. Ganser · Marei Kleiner · Maren Gaese · Holger von der Lippe $(D$

MSB Medical School Berlin, Berlin, Deutschland

\section{Indizierte Prävention} hochstrittiger Trennungseltern: eine qualitative Implementationsstudie zu „Kinder aus der Klemme"
Das indizierte Präventionsprogramm „Kinder aus der Klemme“ (KadK) wird seit einigen Jahren europaweit angeboten und richtet sich an Eltern, die in einer hochstrittigen Trennungssituation verhaftet sind [22, 23]. Besondere Merkmale von KadK stellen das multifamilientherapeutische Konzept, d.h. die parallele Arbeit mit mehreren Elternpaaren, ihren Kindern und Netzwerkpersonen aus derselben Familie sowie die avisierte Kombination von systemischen und gruppentherapeutischen Wirkfaktoren dar. Zwei Berliner Träger gehörten seit 2018 zu den geförderten Einrichtungen mit diesem Angebot in der Stadt.

Der vorliegende Beitrag skizziert die wissenschaftliche Implementationsforschung durch die Medical School Berlin von 2018 bis 2020. Dabei werden Ergebnisse qualitativer Interviews präsentiert, welche die Erfahrungen der Therapeut:innen, Eltern, Kinder und Netzwerkpersonen mit der Implementation des Programms aufzeigen.

\section{Hintergrund}

\section{Das Phänomen hochstrittiger Trennungen von Eltern}

Die Trennung von Eltern stellt in westlichen Industriegesellschaften ein kritisches Familienereignis dar, welches nach einer Phase mit erhöhtem Stress und Konflikten von allen Beteiligten schließlich meist gut bewältigt werden kann.
Aus dem alten Drama einer Scheidung ist heute in den meisten Fällen ein pragmatischerer Übergang geworden, der mit Begriffen wie „familialer Reorganisation“ oder „Binuklearisierung der Familie“ beschrieben wird [10, 17].

Jedoch gelingen diese Reorganisation und Bewältigung bei einem Teil der Trennungseltern auf Dauer nicht. Diese bleiben bisweilen über Jahre oder Jahrzehnte in erbitterten Streitigkeiten mit hohem (offenem wie unterschwelligem) Konfliktniveau sowie juristischen Auseinandersetzungen verhaftet. Diese Trennungseltern werden als hochstrittig („high-conflict separations“, HCS) bezeichnet. Das zentrale Definitionsmerkmal ist dabei das Konfliktniveau der Expartner:innen, welches erheblich über das normale zeitliche wie soziale Maß hinausgeht. Schätzungen zufolge verlaufen etwa $5 \%$ von elterlichen Trennungen in Deutschland derart hochkonflikthaft. Damit sind jährlich schätzungsweise bis zu 15.000 Kinder von einer solchen hochstrittigen Trennung ihrer Eltern neu betroffen (Jahresinzidenz [5, 7]). Da sich jeder Einzelfall mit Hochstrittigkeit aber über einen längeren Zeitraum erstreckt, gehen einzelne Autor:innen sogar von kumuliert 50.000 aktuell betroffenen Kindern in Deutschland aus (Punktprävalenz [7]).

Die differenziellen Entwicklungsfolgen einer elterlichen Trennung - dies ist seit Längerem bekannt - hängen für beteiligte minderjährige Kinder v. a. vom
Konfliktniveau der Trennung ab [1, 8, 10, 17]. Daher leiden Kinder im Fall einer hochstrittigen Trennung ganz besonders. Studien zeigen, dass andauernde emotionale Belastungsgefühle im Familienkontext externalisierendes wie internalisierendes Problemverhalten, eine verringerte Leistungsfähigkeit und psychische Symptome bei den betroffenen Kindern auslösen können [1,9,26]. Bisweilen werden die Kinder auch in den Kampf der Eltern direkt hineingezogen oder funktionalisiert und ihre Entwicklung sowie ihr Wohlbefinden geraten für die Eltern völlig aus dem Blick [12, 13, 24, 26]. Es besteht Präventionsbedarf.

\section{Indizierte Prävention bei \\ Hochstrittigkeit und das \\ Programm „Kinder aus der \\ Klemme" (KadK)}

Wie hoch konflikthaften Trennungseltern $^{1}$ am besten geholfen werden kann, wird in der Literatur uneinheitlich diskutiert. Einerseits findet Fichtner [9], dass auch hochstrittige Eltern am ehesten von klassischer Einzelberatung profitieren können - in seiner Studie zu etwa

\footnotetext{
1 Hier und im Folgenden verwenden wir den vereinfachenden Sammelbegriff "Trennungseltern" für zwei getrennte Personen mit gemeinsamen Kindern - unabhängig davon, ob es sich um vormals verheiratete oder unverheiratete, gleich-oder gegengeschlechtliche Eltern mit oder ohne Sorgerecht handelt.
} 
Tab. 1 Ausgewählte Inhalte und Ablauf der Arbeit mit den Eltern im Programm „Kinder aus der Klemme"

\begin{tabular}{l} 
Termine \\
\hline Erst- und Infoge- \\
spräch \\
Beide Eltern oder \\
auch einzeln - ohne \\
Kinder \\
Vortreffen \\
Mit einer Tren- \\
nungsfamilie Eltern \\
und deren Kind(er) \\
Netzwerkinfo- \\
abend \\
Alle teilnehmenden \\
Eltern ohne Kinder, \\
mit Netzwerkperso- \\
nen \& Erfahrungs- \\
expert:innen \\
1. Elterngruppen- \\
sitzung
\end{tabular}

Inhalte (Auswahl)

Unverbindliche Vorstellung der Erwartungen und Haltung des Therapeut:innen-Teams. Leitfrage: „Sind Sie bereit mit uns zu untersuchen, was Sie verändern können?", Informationen zum Programm

Nur mit Kindern: Bild der zwei Häuser und des Zwischenraums. Nur mit Eltern: In Einzelgesprächen berichten über ihre Erfahrungen, den größten Schmerz, Tragik und Hoffnung (Verletzlichkeitszyklus) \& ReflectingTeam der Therapeut:innen über das Gespräch mit den Eltern

Wer wir sind und wie wir arbeiten. Schmerz, Tragik, Hilflosigkeit betreffen auch das Netzwerk. Mithilfe des Netzwerks ist bedeutsam, wünschenswert und Teil des Programms: Botschaften an das Netzwerk: „Wir sind auch für Euch ansprechbar, ohne Euch geht es kaum!"

Selbstvorstellung der Eltern mit einer Geschichte über ihr(e) Kind(er), Austausch über drei destruktive Kommunikationsmuster.

Hausaufgaben: Elternarbeitsbuch - Identifikation dieser Muster im Alltag und Austausch mit Netzwerkpartner:innen

2. Elterngruppen- Dialog über Veränderungen, Beobachtungen und Hausaufgaben. Übung sitzung auf Kinderstühlen zum Erfahren der drei Konfliktmuster, Austausch in der Gesamtgruppe über die Folgen für Kinder,

Hausaufgaben: Formulierung eines Trennungsnarrativs, mit dem Kinder leben können, Netzwerk zu Rate ziehen

3. Elterngruppen- Dialog über Veränderungen, Beobachtungen und Hausaufgaben. Übung: sitzung

4. Elterngruppensitzung

5. Elterngruppensitzung

6. Elterngruppensitzung

7. Elterngruppensitzung

8. Elterngruppensitzung

Nachsorgetreffen

Beide Eltern mit Kind(ern)

Anmerkung: Wir stellen hier aus Platzgründen vordergründig die Arbeit mit den Eltern dar (weitere Erläuterungen im Text)
$30 \%$. Gleichlautend berichten Autoren wie beispielsweise Ritzer-Sachs [16], Spengler [19] und Winkelmann [25] von durchaus positiven Erfahrungen mit der herkömmlichen Beratung von hochstrittigen Trennungseltern.

Demgegenüber stehen Autor:innen, die betonen, dass es besonderer Methoden bedürfe, um hochstrittigen Trennungseltern effektiv helfen zu können. Dies liege an Besonderheiten von Hochstrittigkeit wie etwa der besonderen Erbittertheit, Langwierigkeit und juristischen Involvierung bei diesen Eltern. Aufgrund dieser Besonderheiten erlebten es diesen Autor:innen zufolge professionell Helfende allzu häufig, selbst in die destruktiven Dynamik hineingezogen oder funktionalisiert zu werden und in einen Zustand von eigener Hilflosigkeit, Frustration und Erschöpfung zu geraten („failure“, [11], S. 189). Auch die individuelle Beratung oder Therapie mit den Kindern könne hier geringe Erfolge verzeichnen, da der Konflikt der Eltern durch die Arbeit mit den Kindern nicht verbessert werde $[6,11,21]$. Diese konträren, hier in aller Kürze skizzierten Ansichten in der Literatur sind bislang nicht abschließend beantwortet und müssen daher bis auf Weiteres als gleichwertig betrachtet werden.

Von Autor:innen der letztgenannten Position wurden in den vergangenen Jahren indizierte Präventionsprogramme speziell für das Phänomen der Hochstrittigkeit entwickelt. Das erste im deutschsprachigen Raum war der seit den 2000er-Jahren durch das Deutsche Jugendinstitut konzipierte und inzwischen weithin angebotene strukturierte Elternkurs „Kinder im Blick“ [13, 24]. In diesem Angebot nehmen hochkonflikthaft getrennte Eltern an mehreren separaten Mütter- und Vätergruppen teil. Die Evaluation zeigt erste positive Wirkungen [24], wobei eine umfangreiche Wirksamkeitsstudie aktuell in Arbeit ist [2].

Einen etwas anders fundierten Ansatz stellt seit einigen Jahren ein in den Niederlanden entwickeltes, multifamiliengruppentherapeutisches Programm dar, welches inzwischen europaweit angeboten wird [22, 23]. Das Programm „Kinder aus der Klemme“ (KadK; engl. 
Präv Gesundheitsf 2023 · 18:68-77 https://doi.org/10.1007/s11553-022-00932-6

(c) Der/die Autor(en) 2022

\section{A. Ganser · M. Kleiner · M. Gaese · H. von der Lippe}

\section{Indizierte Prävention hochstrittiger Trennungseltern: eine qualitative Implementationsstudie zu „Kinder aus der Klemme"}

\section{Zusammenfassung}

Hintergrund. Hochstrittige Trennungen der Eltern stellen ein erhebliches Risiko für die Entwicklung von Kindern dar. Das spezifische Gruppenprogramm „Kinder aus der Klemme" basiert auf einem multifamilientherapeutischen Konzept und zielt darauf ab, bisherige Konfliktmuster zugunsten positiver Elternfunktionen zu verändern. Die vorliegende Implementationsstudie untersucht, ob und wie das Angebot in seiner deutschsprachigen Adaptation von den Nutzern und Therapeut:innen als hilfreich erfahren wird und wie das Angebot potenziell optimiert werden kann.

Methodik. Es wurden qualitative Interviews mit $n=34$ Beteiligten (darunter Trennungseltern, Kinder, Netzwerkpartner:innen und
Therapeut:innen) eines neu implementierten Berliner Angebotes des Programms untersucht. Die Auswertung erfolgte per Qualitativer Inhaltsanalyse.

Ergebnisse. Alle Befragten berichten eingehend über die besonderen Herausforderungen während des Einstiegs in das Programm. Zahlreiche positive und bereichernde sowie einige konkret verbesserungswürdige Aspekte des Programms werden von allen BefragtenGruppen wahrgenommen. Insbesondere gruppentherapeutische (z. B. Kohäsion, Beobachtungslernen) wie systemische Effekte (z. B. Ressourcenaktivierung, Stärkung der Elternfunktionen) lassen sich aus den Interviews für das Programm rekonstruieren.
Diskussion. „Kinder aus der Klemme“ stellt eine ansprechende und subjektiv wirksame Intervention für hochkonflikthafte Trennungseltern und ihre Kinder dar. Auch Familien, die zuvor bereits eine Vielzahl anderer Hilfen in Anspruch genommen haben, können sich darauf gut einlassen. Zukünftig könnte aus Sicht der Befragten die Dauer des Kurses erweitert werden, und auch die Arbeit mit den Kindern und Netzwerkpartner:innen könnte weiterentwickelt werden.

\section{Schlüsselwörter}

Hochstrittigkeit · Multifamiliengruppen · Netzwerkansatz · Elternverhalten · Systemische Effekte

\section{Prevention program for high-conflict separated parents: a qualitative implementation study on "No Kids in the Middle"}

\section{Abstract}

Background. High-conflict separations of parents pose a considerable risk for the development of children. The specific group program "No Kids in the Middle" is based on a multifamily therapeutic concept and aims to change previous conflict patterns in favor of positive parenting functions. The present implementation study examines whether and how the program in its Germanlanguage adaptation is experienced as helpful by users and therapists and how the offer can potentially be optimized.

Methodology. These primary research questions were examined through qualitative interviews with $n=34$ participants (including separated parents, children, network partners, and therapists) of a newly implemented Berlin offer of the program. Qualitative content analysis was used for analysis.

Results. All interviewees reported in detail about the special challenges during their entry into the program. Numerous positive and enriching aspects were perceived by all respondent groups, as well as some concrete issues of the program that need improvement. In particular, group therapeutic (e.g., cohesion, observational learning) as well as systemic effects (e.g., resource activation, strengthening of parenting functions) were reconstructed from the interviews for the program.
Discussion. "No Kids in the Middle" represents an appealing and subjectively effective intervention for high-conflict separation parents and their children. Families who had previously tried a variety of other support interventions became engaged in the current program and felt helped by it. In the opinion of the interviewees, the duration of the course could be extended in the future and the work with the children and network partners could also be further developed.

\section{Keywords}

High-conflict separations - Multiple-family groups - Network approach · Parenting behavior - Systemic effects
„No kids in the middle“, ndl. „Kinderen uit de knel") arbeitet mit beiden Trennungseltern in derselben sich aus insgesamt sechs getrennten Elterndyaden zusammensetzenden Multifamiliengruppe, während für die minderjährigen Kinder zeitgleich eine eigene Gruppe durchgeführt wird. Zudem werden alle teilnehmenden Eltern dazu verpflichtet, wichtige Personen aus ihrem persönlichen Umfeld zu benennen (z. B. andere Familienangehörige, Freunde, neue Part- ner:innen etc.), um diese zur Teilnahme am Programms einzuladen [21]. Denn die Programmentwickler:innen bemerkten in ihrer Arbeit mit hochstrittigen Eltern, dass allzu oft die zwei ebenfalls getrennten Netzwerke der ehemaligen Partner:innen einen erheblichen Beitrag zur Aufrechterhaltung des Konflikts einnehmen können und daher involviert werden sollten [21].

Der Ablauf des Programms KadK ist mit einem Schwerpunkt auf die Eltern- gruppen im groben Überblick in • Tab. 1 dargestellt. In der sog. Elterngruppe treffen sich die sechs Trennungselterndyaden $\mathrm{zu}$ insgesamt acht zweistündigen Gruppensitzungen meist in einem 14tägigen Rhythmus. Diese Gruppe wird von zwei Therapeut:innen angeleitet und beinhaltet eine klare Struktur an therapeutischen Inhalten. Dazu zählen z.B. Rollenspiele, Hausaufgaben, Feedbackrunden, gemeinsame Gruppenaufgaben und Ähnliches [22, 23]. Zeitgleich neh- 
men die minderjährigen Kinder und Jugendlichen der Familie an der sog. Kindergruppe teil, in denen von zwei weiteren Therapeut:innen ein recht freies Kreativprogramm angeboten wird. Dieses soll den Kindern und Jugendlichen - frei von starrem Programm oder Erwartungsdruck - die Möglichkeit bieten, eigenen Gefühlen und Gedanken Ausdruck zu verleihen und sich darüber mit Anderen auszutauschen. Kinder und Eltern bereiten während des Programms zudem jeweils eine kleine Präsentation für die andere Gruppe vor, die sich dann gegenseitig vorgestellt werden $[11,22$, 23].

Die begleitenden Netzwerkpersonen der Eltern, die zu zwei gesonderten Terminen eingeladen werden, können zu Beginn frei gewählt werden und sind als „Mentor:innen“ von besonderer Bedeutung, da sie den Veränderungsprozess begünstigen und flankieren sollen. Van Lawick und Visser [22, 23] führen hierzu aus, dass die Trennung eines Elternpaares eben nicht nur eine Trennung von zwei individuellen Familienmitgliedern darstellt, sondern auch eine weiterreichende Trennung von zwei zuvor verbundenen personalen Netzwerken. Da diese Netzwerke im Trennungsverlauf von hochstrittigen Eltern oft eine negative Position gegenüber den jeweiligen Expartner:innen einnehmen und den Versöhnungsprozess sogar konterkarieren können („Krieg zweier Dörfer“), wird die Einbeziehung dieser Netzwerke für die Intervention als besonders bedeutsam erachtet. Sie werden eingeladen, als „Programmmentor:innen“ für ihre „Mentees“ (also die Trennungseltern) unterstützend und entlastend zur Seite zu stehen. So sollen sie beispielsweise bei den lösungsund ressourcenorientierten Hausaufgaben der teilnehmenden Eltern unterstützen [21].

Die Kindergruppe läuft weniger vorstrukturiert als die Elterngruppe ab. Den Kindern wird es hier freigestellt, sich über schöne und schlimme Erinnerungen, über ihre Erfahrung von Konflikten, Loyalitätsgefühlen und hilfreichen Erfahrungen verbal oder spielerisch (z. B. in Form von kreativen Aktivitäten und Arbeiten an einer Präsentation) auszudrücken. Sie können diese Einladung an- nehmen oder nicht, indem sie beispielsweise Schularbeiten oder Spielen nachgehen. Die Hauptveränderungsarbeit soll in diesem Programm von den Eltern, nicht von den Kindern, geleistet werden. Dazu sind alle Inhalte, Übungen und Hausaufgaben in einem Arbeitsbuch für Eltern niedergeschrieben [20]. Die Grundidee dieser gemeinsamen Arbeit mit mehreren getrennten Eltern, den Kindern und einigen Netzwerkpersonen ist es, dass sich gruppen-, gemeinde- und familientherapeutische Wirkfaktoren möglichst effizient ergänzen sollen.

Voraussetzung für eine Programmteilnahme ist die Bereitschaft beider Trennungseltern, gemeinsam an acht Gruppensitzungen inklusive Vor- und Nachbereitungstreffen aktiv teilzunehmen. Außerdem ist zu garantieren, dass während der Teilnahme alle die Trennung betreffenden gerichtlichen Auseinandersetzungen gestoppt sind und innerhalb der familiären Beziehungen weder Suchtverhalten, Gewalt oder psychotische Krisen die aktuell als hinreichend sicher angenommenen Bedingungen bedrohen.

Die wissenschaftliche Begleitstudie: Ansatz und Fragestellungen

Der Evaluationsstand zu KadK ist aufgrund der Neuartigkeit des Programms noch recht schmal. Die ersten empirischen summativen Evaluationsergebnisse sowie klinischen Berichte aus der niederländischen Implementation heraus sprechen für eine grundlegende Wirksamkeit des Programms [18]. Weitere Outcome-Studien sind derzeit international in Vorbereitung, werden aber noch durch die kleinen Gruppengrößen und die geringe Forschungsförderung für diese Arbeit erschwert - Gründe, die auch im vorliegenden Fall der Implementation des Berliner Programms die Wirksamkeitsforschung noch ausschlossen. Dies begründete die Wahl eines formativen und qualitativen Evaluationsansatzes.

Bei diesem Ansatz geht es um die Erfassung von Durchführungscharakteristika einer Intervention aus den Perspektiven von Teilnehmenden im Hinblick auf ihre mögliche Weiterentwicklung, Anpassung oder Optimierung [3, 15].
Durch den methodischen Fokus auf die Perspektiven von Nutzer:innen und Anbieter:innen eines Programms (also hier die hochstrittigen Trennungseltern, deren Kinder und Netzwerkpartner:innen sowie die Therapeut:innen) soll rekonstruiert werden, in welcher Weise ein Programm gestaltet und genutzt wird, was die Einsatz- und Einflussmöglichkeiten mit bestimmt. Patton [15] bezeichnet dies als die personenbezogenen Faktoren der formativen Evaluation und plädiert dafür, diese im Sinne des zu rekonstruierenden subjektiven Einsatzund Anwendungsnutzens für die mögliche Weiterentwicklung als bedeutsam $\mathrm{zu}$ erkunden. Wirksamkeit (im Sinne der summativen Evaluation) ist hier allenfalls ein Randaspekt.

Mit dem Ziel einer formativen Implementationsstudie [3] zu den ersten vier Programmdurchläufen eines neuen Berliner Angebots wurde eine Forschungsgruppe, welche personell, räumlich und informationell unabhängig vom Therapeut:innen-Team arbeitete, unter der Leitung des Letztautors zu einer Begleitstudie beauftragt. Die teilnehmenden Trennungsfamilien bei dem neuen Berliner Angebot rekrutierten sich ausschließlich aus starken Empfehlungen von Jugendämtern in Kooperation mit Fachdiensten (z. B. EFB, KJPD) und/oder familiengerichtlichen Verfahrensbeiständen heraus. Alle Familien konnten seit mehreren Jahren und trotz verschiedener anderer Versuche (z. B. Beratung, Gerichtsverfahren, „Kinder im Blick“ etc.) ihren Streit bislang nicht befrieden.

Die leitenden explorativen Fragestellungen der Begleitstudie lauteten:

a) Welche Erfahrungen berichten alle relevanten Personengruppen (Eltern, Kinder, Netzwerkpartner:innen und Therapeut:innen) mit dem Programm KadK, v. a. im Hinblick auf den eigenen Einstieg, die konkrete Umsetzung und die subjektiven Effekte (subjektive Nützlichkeit) des Programms?

b) Welche Wünsche und Vorschläge zu einer möglichen Weiterentwicklung des Programms werden von den relevanten Personen geäußert? 


\section{Originalarbeit}

\begin{tabular}{llll}
$\begin{array}{l}\text { Tab. 2 } \\
\text { Befragtengruppe }\end{array}$ & Die Datengrundlagen und die qualitative Inhaltsanalyse im Überblick & \\
\hline Interviews $(\boldsymbol{n})$ & $\begin{array}{l}\text { Interviewdauer } \\
\text { (min; Range, [min-max]) }\end{array}$ & $\begin{array}{l}\text { Haupt- } \\
\text { kategorien (n) }\end{array}$ \\
\hline Therapeut:innen & 8 & $15-90$ & 7 \\
\hline Trennungseltern & 13 & $35-120$ & 10 \\
\hline Netzwerkpartner:innen & 6 & $40-100$ & 8 \\
Kinder & 7 & $20-50$ & 4 \\
Gesamt & 34 & $15-120$ & 29
\end{tabular}

\section{Methodik}

\section{Stichprobe}

Die Gewinnung der Befragungsstichprobe erfolgte - wie in vergleichbaren Studien auch [11] - auf freiwilliger Basis unter allen Beteiligten aus vier Durchläufen des Programms. Die Studienteilnehmenden wurden durch die persönliche Ansprache von Programmhospitantinnen aus der Forschungsgruppe über die Studie informiert und zu persönlichen Interviews eingeladen. Insgesamt wurden so durchgangsübergreifend 44 Trennungseltern sowie 10 Therapeut:innen angesprochen. Der Kreis der Teilnehmer:innen umfasste Eltern, deren Trennung als Paar zwischen 1 und 10 Jahren zurücklag, was bei allen Paaren auch gleichlautend mit der Dauer des Konflikts war. Alle Eltern besaßen Erfahrungen mit Trennungsberatungen, Mediationen oder familiengerichtlichen Verfahren, bei jedem Trennungspaar waren 1-3 Kinder im Alter von 4,5 bis 14 Jahren sowie 1-3 Netzwerkpartner:innen involviert. Bei den Therapeut:innen handelte es sich um Sozialpädagog:innen und Psychotherapeut:innen, die alle an einer spezifischen systemisch-therapeutischen Fortbildung zu Kinder aus der Klemme teilgenommen hatten und über Erfahrungen in der Arbeit mit Kinder, Familien und Multifamiliengruppen verfügten.

Es konnten schließlich insgesamt 34 leitfadengestützte Interviews realisiert werden, davon $n=13$ mit Trennungseltern (6 Mütter), $n=7$ mit Kindern im Alter von 6 bis 13 Jahren (3 Mädchen), $n=8$ Therapeut:innen (2 Männer) und $n=6$ Netzwerkpartner:innen (5 Frauen), wovon 3 neue Partner:innen und 3 enge Freund:innen der Teilnehmenden waren.

\section{Instrumente und methodisches Vorgehen}

Die problemzentrierten Interviews [27] mit den Therapeut:innen fanden zu verschiedenen Zeitpunkten in Bezug auf einen Programmdurchgang (vor, während und nach einem Durchgang) statt, um die individuelle Vorbereitung, die aktuellen Erfahrungen sowie den Rückblick auf den Gesamtdurchgang rekonstruieren zu können. Die Interviews mit den teilnehmenden Eltern, den Kindern und den Netzwerkpartner:innen erfolgten zum Ende eines Programmdurchgangs, um einen möglichst vollständigen Erfahrungsbericht zum Programm erfragen zu können.

Die in den Interviews eingesetzten Leitfäden fokussierten insbesondere auf:

- die Erwartungen und Vorerfahrungen der Personen zu Programmbeginn,

- die Erfahrungen während des Programmbeginns und die eigenen Erfahrungen während des gesamten Programmdurchgangs sowie

- die Wünsche und ungedeckten Bedürfnisse der Befragten bezüglich des Programms.

\section{Analytisches Vorgehen}

Alle qualitativen Interviews wurden aus der Audioaufnahme wie gehört transkribiert. Gemäß der strukturierenden und der zusammenfassenden Inhaltsanalyse nach Mayring [14] wurden in einem deduktiv-induktiven Wechselspiel aus Vorannahmen und neuen Informationen in gemeinsamen Interpretationsteams der Forschungsgruppe umfangreiche Kategoriensysteme entwickelt. In diesen Kategoriensystemen wurde das von den Befragten Gesagte zusammengefasst und abstrahiert, wobei sich je nach Daten- grundlage zwei bis drei Abstraktionsebenen aus Haupt-, Unter- und UnterUnterkategorien ergaben. Die Zahl der entwickelten Hauptkategorien variierte schließlich zwischen 4 und 10 pro Befragtengruppe. - Tab. 2 stellt diese Kategorisierung im Überblick vor. Die erarbeiteten zentralen Kategorien werden für die Beantwortung der Forschungsfragen in der folgenden Ergebnisdarstellung durch Kursivsetzung berichtet.

\section{Ergebnisse}

$J a$, es geht nach oben, (..) ein bisschen. (...). Aber was wir, denke ich, auf jeden Fall also ich auch gelernt habe, das Wichtigste ist eben, friedvoller miteinander umzugehen, es zu versuchen, nicht eskalieren zu lassen, was ja nicht immer so ganz gelingt bislang, und dass es vor allem auch für unser Kind sehr wichtig ist, dass es da mehr Frieden zu Hause hat, mehr Stabilität und nicht das Gefühl hat, zwischen uns (...), zwischen dem Konflikt (zu stehen). (KursI_Va1)

Subjektive Sicht auf den Einstieg, die Umsetzung und die Effekte von KadK

\section{Therapeut:innen}

Ein innovatives Präventionsprogramm für eine womöglich anspruchsvolle Zielgruppe verlangte oft auch von den Therapeut:innen den „Sprung ins kalte Wasser“ (Haupt- und Unterkategorien der qualitativen Inhaltsanalyse im Folgenden kursiv). Dabei reichten die Vorerwartungen der Therapeut:innen auf das neue Präventionsprogramm von Vorfreude bis hin zur Erwartung, besonders herausfordernde Situationen zu erleben. Auf ihre ersten Gruppensitzungen bereiteten sich die Therapeut:innen vor, indem Sie sich auf ihre systemische Grundhaltung, die Vorerfahrungen in der Arbeit mit Paaren und Familien sowie die programmspezifische Fortbildung zu KadK bezogen („Wissensfundus“). Dazu zählten auch die spezialisierte Fortbildungsliteratur und die Vorbesprechungen im Therapeut:innen-Team vor Programmstart.

Im Hinblick auf die Erfahrungen bei der Umsetzung des Programms betonten 
die Therapeut:innen, dass sie in der Tat von ihrer systemischen Grundausbildung und dem unterstützenden kollegialen Austausch im Team besonders profitieren konnten. Diese Ressourcen schützten den Therapeut:innen zufolge vor den besonderen Anforderungen der Arbeit mit Hochstrittigen und so erlebten sie die Arbeit im Programm als sinnvoll und bereichernd. Hierbei schätzten die Therapeut:innen der Elterngruppe die in der Fortbildung erlernten strukturierten Gruppenübungen (wie z. B. Rollenspiele und Feedback-Techniken) als wirksame Tools, um die Eltern zur gemeinsamen Arbeit zu bewegen und darin zu führen.

\section{Trennungseltern}

Auf Seiten der Trennungseltern gab es während der Phase des Einstiegs in das Programm zunächst Ängste und Skepsis bezüglich dessen, was sie wohl erwarten würde. Bisweilen kamen die Eltern mit polaren Selbstbildern entweder des Opfers (der Umstände) vs. des Könners (i.S.e. subjektiven Meisterschaft der Situation) in die Gruppen, woraus sich die je individuellen Befürchtungen (nämlich der Über- vs. Unterforderung) begründeten. Diese Sorgen reduzierten sich im Verlauf jedoch und wandelten sich zu einer insgesamt positiven Erfahrung des Programms. Wesentlich für die Eltern waren hierfür der Anstoß zur Selbstreflexion durch das Erkennen von dysfunktionalen Kommunikationsmustern und das angeleitete Hineinversetzen in die Perspektive ihrer Kinder. In den Elterninterviews standen Erzählungen von einem Aufbrechen des eigenen Gefühls der Isolation mit der Problematik und des gemeinsamen Lernens mit und von den anderen Trennungseltern im Vordergrund.

Wesentlich für diese positiven Entwicklungen war für die Befragten initial, in welchem Ausmaß die Therapeut:innnen auf die individuellen Voraussetzung von (s.oben) bzw. die sporadischen Störungen durch Teilnehmende (z.B. Regelverstöße, Distanziertheit zu Übungen) eingingen und diese behandeln konnten. Eine gelingende Gruppenatmosphäre und -kohäsion erwiesen sich dabei als das zentrale Schlüsselelement, welches zur individuellen Vernet- zung mit Anderen und dem Gefühl einer aufSympathie gründenden Unterstützung für und durch Andere führte. Bisweilen wurde sogar von beginnendem Wohlwollen und Akzeptanz dem:r Expartner:in gegenüber berichtet.

Als einen letzten wichtigen Punkt thematisierten die befragten Eltern eine Besonderheit von KadK, nämlich die Möglichkeit, sich als gesamte Kernfamilie in den Pausenzeiten vor und während einer Gruppensitzung im geschützten Raum der Einrichtung zu sehen. Darin erfahrene Elternpaare hoben dies sogar explizit im Vergleich mit „Kinder im Blick“ positiv hervor - als eine insgesamt seltene, aber schöne Erfahrung. Diese Pausenzeiten wurden aber auch in einen wichtigen Zusammenhang mit der bereits genannten Vernetzung mit anderen Teilnehmenden oder den informellen Gesprächen mit Therapeut:innen genannt. Im Kontakt zum gesamten, aus vier Personen bestehenden Therapieteam schätzten die Eltern insbesondere das durch dieses frei gestaltete Beziehungsangebot und die zugängliche und empathische Haltung.

Das ist so das, was ich hier eigentlich mitnehme, es ist ein Perspektivwechsel vor allem Dingen für mich. Also ein Stück raus aus dem, was ich bisher immer gesehen habe, wie ich es gesehen habe und auch nochmal eine neue Zentrierung doch auch auf [Name des Kindes]. Das ist so das, was ich ganz stark mitnehme. Was ich auch mitnehme ist, ähm, ich bin verantwortlich für meine Eskalation, also da ist [Name des Expartners] nicht verantwortlich für. Das liegt ja in meiner Macht, ob ich die Spirale mache oder nicht, also das ist wirklich nur meine Verantwortung. (KursI_Mu2)

Also ich fand's auch hoch interessant ... die Dynamiken der anderen Paare zu sehen. Manche sehr ähnlich wie man selber, manche ganz anders. Auch zu sehen, was möglich ist, was bei uns noch nicht möglich ist, aber wo man gerne hinkommen würde. Also ich fand's total schön, also auch wenn ich viel geweint habe, weil mich das so ergreift, dann mitfühlen $z u$ können, einfach zu sehen, was möglich ist und wie die Bereitschaft dann da ist von den anderen, aufeinander zuzugehen. (KursIII_Mu3)

\section{Netzwerkpartner:innen}

Die Berichte der befragten Netzwerkpartner:innen erschienen vergleichsweise ambivalent. Typisch waren hier zunächst Schilderungen, dass die eigene einzunehmende Rolle sehr wohl verstanden und oft mit Engagement, Motivation und Optimismus ausgefüllt wurde. Das teilnehmende Elternteil wurde dann in Form einer gemeinsamen Reflexion und der Bearbeitung von Hausaufgaben unterstützt. Als entscheidendes Moment hierfür berichteten die Befragten die Initiative und das Engagement ihrer „Mentees“ (also der Trennungseltern), von denen sie intensiv in die Erfahrungen und Erkenntnisse aus der Elterngruppe involviert wurden und von denen sie beispielsweise auch die Hausaufgaben und die neuen Ideen (wie z. B. die Konzepte der Dämonisierung des Expartners oder der zerstrittenen Dörfer) aktiv mitgeteilt bekamen.

In anderen (wenigen) Erzählungen geschah aber genau das Gegenteil desselben: Die Mentees übernahmen keine aktive Involvierung der Netzwerkpartner:innen, woraufhin sich diese zunächst in einer ambivalenten Haltung bzgl. ihrer Rolle und dem Programm gegenüber sahen und später in Bezug auf die Erfolgsaussichten sogar resignierten. Ganz ähnlich erging es Netzwerkpartner:innen in dem Fall, in dem ihr "Mentee“ von Störungen oder eigenen Distanzierungen in der Gruppe berichtete. Hier verharrten dann auch die Netzwerkpartner:innen in einer kritischen oder ablehnenden Einstellung gegenüber dem Kurs, was sich in eigenen Beiträgen zur Aufrechterhaltung oder gar Verhärtung des Trennungskonflikts äußerte und darüber die Programmziele in Einzelfällen gar unterlief.

Diese Erzählungen ließen sich in der Inhaltsanalyse durch den Begriff des Trennungseltern-Gatekeepings zusammenfassen. Weil die Netzwerkpartner:innen in dieser Fassung des Programms nur 2-mal (bei den Netzwerktreffen) angesprochen und informiert wurden, blieben ihre Sicht auf und ihre Beiträge zu KadK in sehr starkem Maße von den Trennungseltern (ihren Mentees) abhängig. Sie erlebten sich als hilfreiche und motivierende Person, wenn dies genauso von den Paten transportiert 
wurde; und genau umgekehrt, wenn das Gegenteil der Fall war. Wir werden im Kapitel zu den Programmwünschen und in der Diskussion noch einmal auf dieses Phänomen zurückkommen.

\section{Kinder und Jugendliche}

Für die teilnehmenden Kinder und Jugendlichen wurde der Einstieg in das Programm zunächst besonders durch die alterstypischen Befürchtungen wie einem möglichen „Nicht-Hineinpassen“ beschrieben. In diesem Zusammenhang fiel ihnen zu Beginn besonders auch die große Altersheterogenität der Gruppe auf. Die zwanglose und offen gehaltene Struktur der Kindergruppe, die diesen ein Maximum an Gestaltungsfreiheit bot, empfanden die Kinder als positiv sie konnten sich auf den Spaß etwa im gemeinsamen Spiel mit den Anderen oder mit den kreativen Beschäftigungsangeboten gut einlassen. Strukturiertere Elemente, wie etwa die Vorbereitung einer kleinen „Präsentation“ für die Elterngruppe (Sitzung 6), erlebten manche Kinder und Jugendliche dem gegenüber bisweilen sogar als Druck.

Nach der Einstiegsphase wurde dann subjektiv der individuelle Nutzen der vielfältigen Möglichkeiten zu lernen und sich einzubringen thematisiert. Der Austausch mit den anderen Kindern und der sich einstellende Zusammenhalt der Gruppe waren für die Kinder offenbar eine schöne und stärkende Erfahrung. Besonders gerne tauschten sich die Kinder mit Altersgleichen in einer ähnlichen Lebenssituation aus, sodass bisweilen auch neue Freundschaften geschlossen werden konnten. Gerade die älteren Kinder erzählten explizit von einem gestärkten Selbstwertgefühl und Zutrauen durch die Erfahrung in der Gruppe eine gehaltvolle und gute Zeit erlebt zu haben.

Einen ganz besonderen Beitrag leistete dabei auch für die Kinder und Jugendliche die Erfahrung ihre Eltern wieder einmal während des Programms gemeinsam gesehen und Zeit mit beiden verbracht $\mathrm{zu}$ haben. $\mathrm{Zu}$ dieser positiven Erfahrung gehörte für sie auch das freie Angebot, den Therapeut:innen der Kindergruppe konkrete Fragen zur Trennung ihrer Eltern stellen zu können. Insgesamt berichteten die Kinder und Jugendlichen, dass sie mit der Zeit zu einem wirklich positiven Grundgefühl in der Kindergruppe gelangten (bis hin zu einem regelrechten Abschiedsschmerz beim Programmende). Dazu trugen v.a. die in der Gruppe mögliche Selbstöffnung und auch das verbesserte elterliche Verhalten ihnen gegenüber bei.

\section{Wünsche an eine zukünftige Veränderung des Programms KadK}

\section{Therapeut:innen}

Auf die Fragen nach den Optimierungswünschen an das Programm standen für die Therapeut:innen die Weiterentwicklung der Netzwerkarbeit und der Arbeit in der Kindergruppe im Fokus. Man könnte hier, so die geteilten Ideen, die Netzwerkpartner:innen viel stärker in Form von Co-Berater:innen einbeziehen bzw. den Kindern altersspezifischere Angebote in der Gruppe als bisher unterbreiten. Eine verstärkte Kommunikation und Erläuterung über den Sinn und Zweck von Kindergruppe und Netzwerkarbeit könne hier möglicherweise kurzfristig bereits hilfreich sein. Ferner könnte zukünftig der Zeitaufwand präziser eingeschätzt werden. Hierzu wurde angemerkt, dass die individuellen Nachfragen seitens der Teilnehmer:innen zeitlich unterschätzt wurden. Man könne aus Sicht der Therapeut:innen zukünftig beispielsweise über eine moderate Verlängerung des Programms oder das Angebot von einmaligen Einzelterminen für Eltern und Kinder denken.

\section{Trennungseltern}

Für die Eltern stand der Wunsch nach einer ausführlicheren Erklärung der dialogischen Haltung der Therapeut:innen auf der Wunschliste, da das weitestgehende Fehlen eines konfrontativen oder autoritären Interventionsstils nicht allen Teilnehmenden zuvor klar war. Bei Eltern, die sich von Therapeut:innen beispielsweise eine klare Benennung des „Schuldigen“ an der eigene Misere erwarteten, wurde durch die für sie überraschende allparteiliche Haltung der Therapeut:innen teilweise auch der Einstieg etwas erschwert. Ebenfalls wurde angeregt, die Hausaufgaben präziser im Kurs zu thematisieren und zu erläutern sowie im Umfang et- was zu reduzieren. Ein ähnlicher Präzisierungs- und Einbeziehungswunsch der Eltern fand sich bezüglich der Netzwerkpartner:innen wieder: Die genauere Aufklärung über die Rolle der Netzwerkpartner:innen und ein aktiveres Einbeziehen dieser in den Kurs wurden hier formuliert.

Der Wunsch, den Kurs zu verlängern, um angestoßenen Veränderungen mehr Raum und Zeit zu geben oder aber regelhaft Nachsorge- oder Einzeltermine anzubieten, war ein weiteres Thema der Eltern und deckte sich mit den Wünschen der Therapeut:innen. Da gerade auch die Treffen in großer Runde in den Pausenzeiten (mit der eigenen Kernfamilie und anderen Gruppenteilnehmenden, s. oben) ein wichtiges Element von KadK für die Eltern war, wurde hier angeregt, die Pausenzeiten $z u$ verlängern und gleichzeitig auch auf Räumlichkeiten zu achten, die ausreichend groß und eingerichtet sind, um in diesen Zeiten für Alle ein Wohlfühl- und Austauschklima zu implementieren.

\section{Netzwerkpartner:innen}

Auf Seiten der Netzwerkpartner:innen standen - entsprechend den oben bereits berichteten Erfahrungen - ganz besonders die Wünsche nach einer stärkeren und direkteren Information und Kommunikation durch die Therapeut:innen bezüglich ihrer Rolle und ihrer Aufgaben im Rahmen des Programmkonzepts im Vordergrund. Auch wurde der Wunsch nach einer größeren Einbindung des Netzwerks im Programm formuliert, z.B. durch einen regelmäßigen Einbezug der Netzwerkpartner*innen bei weiteren festen Terminen oder einem Zwischen- oder Abschlusstreffen für das Netzwerk insgesamt. Offenbar fühlten sich die Netzwerkpartner:innen in der ursprünglichen Fassung des Programms etwas allein gelassen bzw. vom Trennungseltern-Gatekeeping abhängig. Darauf kommen wir in der Diskussion zurück.

\section{Kinder und Jugendliche}

Für die Kinder und Jugendlichen war, wie oben geschildert, besonders die Einstiegszeit in die Gruppe eine subjektiv schwierige Phase. Von daher wurde von 


\begin{tabular}{|c|c|}
\hline $\begin{array}{l}\text { Infobox } 1 \text { Übersicht über } \\
\text { die zentralen subjektiven } \\
\text { Erfolgsfaktoren für den Einstieg in } \\
\text { und die Durchführung von KadK }\end{array}$ & $\begin{array}{l}\text { Infobox } 2 \text { Übersicht über } \\
\text { die zentralen Wünsche der } \\
\text { Befragten (unabhängig von den } \\
\text { Befragtengruppen) }\end{array}$ \\
\hline $\begin{array}{l}\text { Therapeut:innen betonen die Relevanz } \\
\text { einer systemischen Grundausbildung } \\
\text { und Haltung in Kombination mit einer } \\
\text { progammspezifischen Fortbildung; } \\
\text { Teambesprechungen und -Intervisionen } \\
\text { sind weiterhin fundamental. } \\
\text { Trennungseltern fokussieren auf die } \\
\text { Herstellung einer positiven Gruppenatmo- } \\
\text { sphäre, eines Lernfeldes und -kontextes } \\
\text { für individuelle Perspektivwechsel auf } \\
\text { Konfliktdynamiken und die Bedürfnisse } \\
\text { der Kinder; ferner spielen die Vernetzung } \\
\text { mit Anderen und das Zusammentreffen } \\
\text { als Kernfamilie in den Pausen eine große } \\
\text { Rolle. } \\
\text { Netzwerkpersonen thematisieren ihre } \\
\text { eingenommene Rolle in Abhängigkeit }\end{array}$ & $\begin{array}{l}\text { Überlegt werden sollte die Möglichkeit, } \\
\text { das bisherige Programmangebot mo- } \\
\text { derat zu erweitern: Nachsorgen- und } \\
\text { Einzeltermine sowie eine Ausweitung der } \\
\text { Pausenzeiten werden gewünscht. } \\
\text { - Für die Netzwerkpartner:innen sollte } \\
\text { nach einer Möglichkeit für eine verstärkte } \\
\text { Kommunikation oder einen häufigeren } \\
\text { regelhaften Einbezug in das Programm } \\
\text { gesucht werden. } \\
\text { Für die Kindergruppe sollte nach einer } \\
\text { altersentsprechenden Erleichterung } \\
\text { des Einstiegs gesucht werden; mehr } \\
\text { altersgerecht differenzierte Angebote } \\
\text { und der Raum für Einzelgespräche mit } \\
\text { den Therapeut:innen könnten angedacht } \\
\text { werden. }\end{array}$ \\
\hline
\end{tabular}
vom Gatekeeping der Teilnehmenden: sehr positiv, motivierend und hilfreich bis hin zum Gegenteil.

- Kinder und Jugendliche kommen subjektiv durch den Spaß und die zwanglose Angebotsstruktur der Kindergruppe gut an; sie können den Austausch mit Gleichaltrigen und den Therapeut:innen sowie das Treffen mit beiden Eltern in den Pausenzeiten für sich nutzen.

ihnen eine klarere Kommunikation der Zielsetzung der Gruppe (gewissermaßen als Einstiegs- und Vorbereitungshilfe für sie) sowie ein optimierter Umgang mit der hohen Altersspanne (z. B. durch die Teilung der Kindergruppe in Jüngere und Ältere) gewünscht. Auch wurde die Möglichkeit außerhalb der Gruppe Einzelgespräche mit Therapeut:innen führen $\mathrm{zu}$ können als Wunsch geäußert. Dies wurde insbesondere von Kindern und Jugendlichen thematisiert, die ihre eigene Lebenssituation als so speziell und individuell erlebten, dass sie sich - trotz eines vorhandenen Wunsches - nicht trauten, dies in der Gruppe einzubringen. Eine anfänglich betontere Normalisierung von Trennungen der Eltern oder von „besonderen" Lebenssituation (z. B. gleichgeschlechtliche oder nicht Deutsch-muttersprachliche Eltern) durch die Therapeut:innen waren Beispiele für solche von den Kinder und Jugendlichen gewünschten Thematisierungen in der Gruppe oder im Einzelgespräch. die zeigen, inwiefern die Implementationsphase dieses Berliner Angebots des Programms KadK ein ansprechendes und subjektiv anregendes Präventionsprogramm für Familien in hochkonflikthaften Trennungssituationen darstellen kann. Anhand der qualitativ rekonstruierten Erfahrungen von 34 Befragten aus vier Beteiligtengruppen (Therapeut:innen, Trennungseltern, Netzwerkpartner:innen, Kinder und Jugendliche) ließen sich zahlreiche Aspekte aus Teilnehmenden-, aber auch aus Sicht der Therapeut:innen zusammentragen, welche aus subjektiver Sicht der Beteiligten für eine erfolgreiche Implementation des Programms sprechen bzw. diese bedingen.

Interessant - und im Zentrum dieser zusammenfassenden Diskussion - ist zunächst v.a., dass KadK offenbar tatsächlich das „Unmögliche möglich macht“, nämlich hochstrittige Trennungseltern in einem gemeinschaftlichen Raum zur gemeinsamen produktiven Arbeit zu bewegen. Diese, für andere Themen bereits häufig getroffene Beobachtung [4], dass Multifamiliengruppen bei aller Heterogenität der Teilnehmenden gute systemische und gruppentherapeutische Effekte gibt (in dem Fall die hochkonflikthafte Trennungserfahrung), scheint auch aus unseren Ergebnissen heraus zutreffend. Das Programm schafft offenbar einen besonderen Gruppenraum, in dem es gelingt, einen Ort für gemeinsames Lernen, Austausch, Selbstoffenbarung und Selbstreflexion zu bieten. Dies entspricht dem „making space for interaction“ [11, S. 196], welches eine ähnliche norwegische Studie mit Trennungseltern fand. Als veränderndes Moment ragen die gemeinsame Begegnung mit anderen Betroffenen und den anderen Familienmitgliedern sowie die Perspektivwechsel in den Übungen und Rollenspielen hervor. Angesichts der einleitend zitierten Position einer besonderen Herausforderung in der Arbeit mit hochkonflikthaften Trennungseltern ist dies ein unwahrscheinlicher, aber offenbar gelingender Aspekt des Programms. I Infobox 1 stellt die aus unserer Sicht wichtigsten Ergebnisse zusammen.

Auch auf Seiten der Veränderungshaben können, sofern es wenigsten $e i$ ne Gemeinsamkeit aller Teilnehmenden wünsche an das Programm fanden sich hilfreiche Perspektiven in den Interviews, die in Infobox 2 aus unserer Sicht zusammengestellt sind.

\section{Limitationen}

Die Ergebnisse stellen erste Einblicke aus der explorativen Begleitforschung zur Implementation des Programms Kinder aus der Klemme durch zwei Berliner Jugendhilfeträgern dar. Die explorative Natur dieser Forschung macht es notwendig, diese lediglich als erste Anhaltspunkte $\mathrm{zu}$ betrachten und als Beitrag für zukünftige Anschlussund Erweiterungsprojekte zu verstehen. Dazu wurden erste Vorschläge vorgelegt. Die Ergebnisse beanspruchen keine statistische Repräsentativität und sind kategorial allenfalls dann auf die Implementation anderer Angebote beziehbar, wenn diese derselben Programmstruktur und Rekrutierung der Teilnehmenden folgen.

\section{Ausblick}

Insgesamt erweist sich das vorgestellte induktive Studienkonzept als geeignet, um die Beteiligtenperspektive sowie die Wei- 
terentwicklungsmöglichkeiten des Präventionsprogramms zu explorieren. So werden zentrale Verbesserungsimpulse von allen Beteiligten (Therapeut:innen, Eltern, Kinder, Netzwerkpartner:innen) genannt, die die Wirksamkeit des Programms steigern können. Die gewonnenen Erkenntnisse sollten auch in künftige Programme und Studien implementiert und anschließend auf ihren inkrementellen Beitrag zur Wirksamkeit analysiert werden. Insbesondere bieten die noch nicht ausreichend genutzten Potenziale der Netzwerke und Kindergruppen Raum für eine Wirksamkeitssteigerung der Intervention. Als innovatives Programm obliegt es den Forschenden und den Therapeut:innen, es stetig weiter $\mathrm{zu}$ evaluieren und entsprechend anzupassen. Wirksamkeitsstudien im Sinne einer summativen Evaluation werden zukünftig einen nächsten natürlichen Schritt in den Evaluationsbemühungen darstellen.

\section{Fazit für die Praxis}

Das Programm „Kinder aus der Klemme" (KadK) bewirkt aus Sicht der Teilnehmenden eine Verbesserung des Konfliktverständnisses, des Elternverhaltens und der kindlichen Stimmung. Für die Anwendung und Weiterentwicklung von KadK werden folgende Anpassungen empfohlen (siehe auch - Infobox 1 und 2 oben):

- Therapeutenseitig empfiehlt sich eine systemische Grundausbildung, vorangehende Erfahrungen in der Arbeit mit Gruppen und eine programmspezifische Fortbildung.

- Programmseitig ist eine moderate Erweiterung der Pausenzeiten (für die Vernetzung) und der Interventionsdauer (um Haltung und Arbeit der Therapeut:innen detaillierter zu erläutern) zu erwägen.

- Es wird eine Intensivierung der Arbeit in der Kindergruppe angeraten, damit individuelle Erwartungen und schambesetzte Themen besser adressiert werden können.

- Die Netzwerkarbeit zahlt sich aus. Hier ist ein stärkerer Einbezug der Netzwerkpartner:innen zu erwägen.

\section{Korrespondenzadresse}

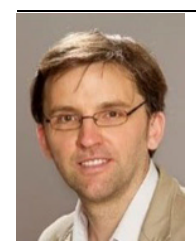

Prof. Dr.

Holger von der Lippe

MSB Medical School Berlin

Rüdesheimer Str. 50, 14197 Berlin, Deutschland

Holger.vonderLippe@ medicalschool-berlin.de

Danksagung. Wir danken den zahlreichen Absolvent:innen und Kolleg:innen der MSB, die zu unterschiedlichen Phasen dieser Studie wichtige Beiträge geleistet haben: M. Bock, A. Jöde, H. Nienhaus, L. Pfeiffer, L. v. Schwander, M. Süßmilch und A. Tursch. Außerdem gebührt dem gesamten Berliner Therapeut:innen-Team unser Dank für den Mut und die Unterstützung dabei, einen forschenden Einblick in ihre Arbeit zu gewähren. C. Bründlinger und Ch. Klein seien als Projektleitende stellvertretend für alle genannt, vielen Dank!

Funding. Diese Implementationsstudie wurde durch die SKala-Initiative finanziell gefördert.

Funding. Open Access funding enabled and organized by Projekt DEAL.

\section{Einhaltung ethischer Richtlinien}

Interessenkonflikt. M.A. Ganser, M. Kleiner, M. Gaese und $\mathrm{H}$. von der Lippe geben an, dass kein Interessenkonflikt besteht.

Für diesen Beitrag wurden von den Autoren keine Studien an Menschen oder Tieren durchgeführt. Für die aufgeführten Studien gelten die jeweils dort angegebenen ethischen Richtlinien.

Open Access. Dieser Artikel wird unter der Creative Commons Namensnennung 4.0 International Lizenz veröffentlicht, welche die Nutzung, Vervielfältigung, Bearbeitung, Verbreitung und Wiedergabe in jeglichem Medium und Format erlaubt, sofern Sie den/die ursprünglichen Autor(en) und die Quelle ordnungsgemäß nennen, einen Link zur Creative Commons Lizenz beifügen und angeben, ob Änderungen vorgenommen wurden.

Die in diesem Artikel enthaltenen Bilder und sonstiges Drittmaterial unterliegen ebenfalls der genannten Creative Commons Lizenz, sofern sich aus der Abbildungslegende nichts anderes ergibt. Sofern das betreffende Material nicht unter der genannten Creative Commons Lizenz steht und die betreffende Handlung nicht nach gesetzlichen Vorschriften erlaubt ist, ist für die oben aufgeführten Weiterverwendungen des $\mathrm{Ma}$ terials die Einwilligung des jeweiligen Rechteinhabers einzuholen.

Weitere Details zur Lizenz entnehmen Sie bitte der Lizenzinformation auf http://creativecommons.org/ licenses/by/4.0/deed.de.

\section{Literatur}

1. Amato PR, Cheadle J (2005) The long reach of divorce: divorce and child well-being across three generations. J Marriage Fam 67(1):191-206

2. Amberg S (2019) Wirksamkeit eines Gruppenangebotes für Eltern in Trennung - Kinder im Blick. Postersitzung präsentiert auf: Konferenz der Universitätspädagog*innen in Bayern (KBUP). Ludwigs-Maximilians-Universität, München

3. Baumeister H (2014) Implementationsforschung in der Klinischen Psychologie, Rehabilitationspsychologie und Psychotherapie. Psychol Rundsch 65:150-158

4. Behme-Matthiessen U, Pletsch T (2021) Multifamilientherapie - eine Einführung. In: BehmeMatthiesen U, Pletsch T (Hrsg) Lehrbuch der Multifamilientherapie. Springer, Berlin, S1-14

5. Dietrich PS, Paul S (2006) Hoch strittige Elternsysteme im Kontext Trennung und Scheidung. Differentielle Merkmale und Erklärungsansätze. In: Weber M, Schilling H (Hrsg) Eskalierte Elternkonflikte. Juventa, Weinheim, S13-28

6. Eckhardt-Ringel A (2021) Kinder aus der Klemme - Multifamilientherapie mit hochstrittigen Trennungsfamilien. In: Behme-Matthiesen U, Pletsch T (Hrsg) Lehrbuch der Multifamilientherapie. Springer, Berlin, S107-121

7. Fichtner J (2006) Konzeptionen und Erfahrungen zur Intervention bei hoch konflikthaften Trennungs- und Scheidungsprozessen - Exemplarische Praxisprojekte. https://www.dji. de/fileadmin/user_upload/bibs/Hochstrittige_ Fichtner_2007.pdf. Zugegriffen: 7. Mai 2020 (Online-Publikation des DJI)

8. Fichtner J (2012) Hilfen bei Hochkonflikthaftigkeit? Forschungsergebnisse zu Merkmalen und möglichen Interventionen in belasteten Nachtrennungsfamilien. ZKJ 2:46-54

9. Fichtner J (2019) Hochkonflikthaftigkeit in Familiengerichtlichen Verfahren. In: Volbert $\mathrm{R}$, Huber A, Jacob A, Kannegießer A (Hrsg) Empirische Grundlagen der familiengerichtlichen Begutachtung: Familienpsychologische Gutachten fundiert vorbereiten. Hogrefe, Göttingen, S51-72

10. Fuhrer U (2009) Lehrbuch Erziehungspsychologie, 2. Aufl. Huber, Göttingen, Bern

11. Høigilt A, Bøe T (2021) Doubt, hope, pain, and new discoveries: parents' experiences of the highconflict program 'no kids in the middle. Aust N Z J Fam Ther 42:188-200

12. Kindler H, Walper S, Friedrich-Bäcker V (2017) (Begleiteter) Umgang und Kindeswohl: Eine Forschungsübersicht. In: Klinkhammer M, Engel G (Hrsg) Handbuch begleiteter Umgang. Bundesanzeiger, Köln, S33-67

13. Krey M, Pröls C, Walper S (2013) Der Elternkurs "Kinder im Blick". Ein Programm für Trennungseltern. In: Röhrle B, Christiansen H (Hrsg) Hilfen für Kinder und Jugendliche in schwierigen Situationen. Deutsche Gesellschaft fürVerhaltenstherapie, Tübingen, S235-261

14. Mayring P (2015) Qualitative Inhaltsanalyse: Grundlagen und Techniken, 12. Aufl. Beltz, Weinheim

15. Patton MQ (2011) Essentials of utilization-focused evaluation, 1. Aufl. SAGE, Thousand Oaks

16. Ritzer-Sachs U (2013) Balanceakt Vertrauensschutz. Voraussetzungen und Beispiele gelingenderBeratung im Kontext Hochkonflikt. In:Weber M, Alberstötter $\mathrm{U}$, Schilling $\mathrm{H}$ (Hrsg) Beratung von Hochkonfliktfamilien: Im Kontext des FamFG. Beltz Juventa, Weinheim, S317-328 
17. Schneewind KA (2010) Familienpsychologie, 3. Aufl. Kohlhammer, Stuttgart

18. Schoemaker K, Kruijff A, de Visser M, van Lawick J, Finkenauer C (2017) Vechtscheidingen. Beleving en ervaringen van ouders en kinderen en verandering na Kinderen uit de Knel. Onderzoeksrapport. Academische Werkplaats aanpak Kindermishandeling, Amsterdam, Utrecht

19. Sprengler P (2013) Zum Befrieden destruktiver Elternkonflikte im Interesse der Kinder. Die Lebensflussmethode in der Trennungs- und Scheidungsarbeit. In: Weber M, Alberstötter U, Schilling $\mathrm{H}(\mathrm{Hrsg})$ Beratung von Hochkonfliktfamilien: Im Kontext des FamFG. Beltz Juventa, Weinheim, S41-76

20. van der Elst E, Wierstra J, van Lawick J, Visser M (2020) Kinder aus der Klemme - Arbeitsbuch für Eltern. Carl-Auer, Heidelberg

21. van Lawick J, van der Elst E (2021) Ohne Euch geht es nicht! Netzwerkarbeit bei Trennungskonflikten Eine Notwendigkeit. In: Klein C, Furman B (Hrsg) Die Kraft des Miteinander. Innovative Methoden der Netzwerk- und Gemeinschaftsarbeit in Familien, Therapie, Schule und Beratung. CarlAuer, Heidelberg, S149-174

22. van Lawick J, Visser M (2017) Kinder aus der Klemme: Interventionen für Familien in hochkonflikthaften Trennungen. Carl-Auer, Heidelberg

23. Visser M, van Lawick J (2021) Group therapy for high-conflict divorce: the 'no kids in the middle' intervention programme. Routledge, Oxfordshire

24. Walper S, Retz E (2015) Hochstrittige Eltern in Zwangskontexten: Effekte des Elternkurses Kinder im Blick. Prax Rechtspsychologie 25:61-84

25. Winkelmann A (2013) Ressourcenorientierte Arbeit mit hoch strittigen Trennungseltern. Möglichkeiten und Grenzen. In: Weber $M$, Alberstötter $\mathrm{U}$, Schilling $\mathrm{H}$ (Hrsg) Beratung von Hochkonfliktfamilien: Im Kontext des FamFG. Beltz Juventa, Weinheim, S77-91

26. Winkelmann S (2005) Elternkonflikte in der Trennungsfamilie als Risikobedingung kindlicher Anpassung nach Trennung und Scheidung. Dissertation. Universität, Dortmund

27. Witzel A (2000) Das problemzentrierte Interview. Forum Qual Sozialforsch Forum: Qual Soc Researc $1(1): 1-9$ 